\title{
Spectrum of Bacterial Infections Associated with Diabetic Ulcer Patients
}

\author{
Swatishree Pany ${ }^{1}$ (D), Shibani Kumar Sen ${ }^{2}$, G. Prasanna ${ }^{3}$, \\ S. Pati ${ }^{1}$ and Bibhuti Bhusan Pal ${ }^{1 *}$
}

${ }^{1}$ Microbiology Division, ICMR- Regional Medical Research Centre, Chandrasekharpur, Bhubaneswar - 751 023, Odisha, India.

${ }^{2}$ Diabetic Foot Surgeon, Kanungo Diabetes and Multispecialty Hospital, Bhubaneswar - 751 019, Odisha, India. ${ }^{3}$ Department of Obstetrics \& Gynaecology, Kalinga Institute of Medical Sciences, Bhubaneswar - 751024 , Odisha, India.

\begin{abstract}
Diabetic ulceration is a multi-factorial problem which is responsible for considerable morbidity threatening the health care system. By knowing the clinical profile and bio-burden on diabetic ulcer, it is highly beneficial for health treatment. The purpose of the present study was to evaluate the diversity of major bacterial etiology in diabetic ulcer patients. The different samples like pus, swab, and infected tissues were collected from diabetic ulcer patients aseptically and samples were transported through cold chain to the laboratory. The samples were cultured in nutrient agar, mannitol salt agar, macConkey agar and blood agar. Suspected colonies were biochemically confirmed for the isolation of Staphylococcus aureus, Pseudomonas aeruginosa, E.coli and Klebsiella spp. In total 150 diabetic ulcer patients suffering from diabetic foot ulcers, gangrene, burn cases and accidental ulcer cases were analyzed. However, most of the patients developed mono-microbial infection; S.aureus was the most prevalent microbe in diabetic ulcer cases, which were positive for nucA gene.

Keywords: S. aureus, Diabetic foot ulcer, Etiology
\end{abstract}

*Correspondence: bbpal_rmrc@yahoo.co.in; +91 674-2305638

(Received: December 15, 2020; accepted: March 08, 2021)

Citation: Pany S, Sen SK, Prasanna G, Pati S, Pal BB. Spectrum of Bacterial Infections Associated with Diabetic Ulcer Patients. J Pure Appl Microbiol. 2021;15(2):598-603. doi: 10.22207/JPAM.15.2.01

(C) The Author(s) 2021. Open Access. This article is distributed under the terms of the Creative Commons Attribution 4.0 International License which permits unrestricted use, sharing, distribution, and reproduction in any medium, provided you give appropriate credit to the original author(s) and the source, provide a link to the Creative Commons license, and indicate if changes were made. 


\section{INTRODUCTION}

Diabetes is a major health issue that has reached alarming levels with nearly half a billion people living with diabetes worldwide. At present, 463 million adults are living with diabetes and by 2045 , this will rise to 700 million $^{1}$. The proportion of people with type 2 diabetes is increasing in most countries. Along with the rising prevalence of diabetes, an increase in its complications is also expected. Patients with diabetes are more susceptible to infections due to increased glucose levels and suppressed immune response as well as the neuropathy and decreased blood flow to the extremities that lead to slow-healing wounds². Diabetic foot ulcerations and infections are one of the major medical, social, economic problem and the leading cause of morbidity and mortality, especially in the developing countries like India ${ }^{3}$. It is estimated that around $15 \%$ of diabetic patients develop foot ulcers in their life time. Diabetes accounts for more than $50 \%$ of amputation of which $85 \%$ of lower amputation in diabetes patients are preceded by foot ulcers ${ }^{4}$. The global prevalence of diabetic foot ulcers varies from $3 \%$ in Oceania to $13 \%$ in North America, with a global average of $6.4 \%$.The annual incidence of diabetic foot ulcer (DFUs) or necrosis in diabetic patients is known to be about $2 \%$ to $5 \%$ and the lifetime risk ranges from $15 \%$ to $20 \%{ }^{5}$. Diabetic foot ulcers are most commonly caused by irritated or wounded feet, nerve damage, poor circulation and hyperglycaemia. Unusual swelling, redness, irritation and stenches on one or both feet are common initial symptoms of foot ulcers ${ }^{6}$. Prevalence of diabetic foot ulcer in the clinical population of India is found to be $3.6 \%{ }^{7}$. There are scanty reports available regarding the incidence of different bacterial pathogens associated with DFU in Odisha. So the present study has been envisaged to document the spectrum of bacterial pathogens associated with diabetic ulcer in this region.

\section{MATERIALS AND METHODS Study subjects}

One hundred and fifty patients with diabetes attending general surgery ward for diabetic foot ulcer management at different tertiary care Hospital, Bhubaneswar were included during the study period from November 2019 to March 2020. Patients willing to participate in the study were enrolled. Samples were collected from patients with different grades of wounds/ulcers and gangrene after obtaining written informed consent among the age group of $\geq 18$ years.

\section{Data collection}

Socio-demographic and anthropological data [age, marital status, literacy status, occupation, life style (sedentary/ active), familial history (parents/siblings), reasons for stress, duration and severity of disease, along with type of treatment (oral anti-diabetic/ insulin), etc. were collected from patients with diabetes using standardized questionnaires.

\section{Sample collection}

Samples were collected from patients with diabetes having ulcers, surgical sites with infection and other wounds by needle aspirate method. In case of closed wounds, the skin or mucosal surface were disinfected with $2 \%$ chlorhexidine or $70 \%$ alcohol followed by iodine solution (1-2\% tincture iodine or $10 \%$ solution of povidone-iodine). Prior to specimen collection, removal of iodine with alcohol was done. In case of open wounds, debridement, was thoroughly rinsed with sterile saline prior to collection ${ }^{8}$. Tissue samples were obtained from depth of ulcers and transferred aseptically into labelled sampling vials with sterile saline and processed in the Microbiology laboratory Regional Medical Research Centre, Bhubaneswar.

\section{Microbial analysis}

Samples were streaked on nutrient agar, mannitol salt agar, macConkey Agar (MCA) and incubated aerobically for $18-24 \mathrm{~h}$ at $37^{\circ} \mathrm{C}$. Plates with no growth or comparatively low growth will be re-incubated for another 18-48 $\mathrm{h}$ for isolation of bacteria that require extended incubation. Blood Agar plates were incubated in aerobic conditions. Cultural characteristics, morphological appearances of colonies on selective media, gram staining and standard biochemical tests were performed to characterize the bacteria.

\section{DNA extraction}

DNA was prepared from bacteria as described previously $y^{9}$. In brief, a single colony forming unit(CFU) was suspended in $20 \mu$ l of lysis buffer containing $0.25 \%$ (vol/vol) sodium dodecyl sulfate and $0.05 \mathrm{~N} \mathrm{NaOH}$. After heating for 15 min at $95^{\circ} \mathrm{C}, 180 \mu \mathrm{l}$ of high-performance liquid chromatography-grade $\mathrm{H}_{2} \mathrm{O}$ (GCC Biotech) was 
added and the lysis suspension was stored at $20^{\circ} \mathrm{C}$ for further analysis.

PCR analysis for S.aureus

PCR reaction contained $2 \mu$ l of DNA which was added to $23 \mu \mathrm{l}$ of master mix containing $0.1 \mu \mathrm{l}$ of Taq polymerase, $0.5 \mu \mathrm{l}$ of dNTPs , $0.625 \mu \mathrm{l}$ of primers were used for amplification of the nucA gene (nucA1 5'GCGATTGATGG TGATACGGTT3' and nucA2 5'AGCCAAGCCTTGACGAACTAAAGC3'), 1.5 $\mu \mathrm{l}$ of $\mathrm{MgCl}, 2.5 \mu \mathrm{l}$ of 10X PCR buffer and $17.15 \mu \mathrm{l}$ of water ( $G^{2} C C$ Biotech). PCR was performed using the program including initial denaturation at $95^{\circ} \mathrm{C}$ for 10 mins, followed by 30 cycles of annealing with an temperature of $55^{\circ} \mathrm{C}$ was used for nucA gene amplification. Final extension was carried out for 5 mins at $72^{\circ} \mathrm{C}$. The PCR products were visualized using agarose gel (2\%) electrophoresis. Gel images were captured using the Gel documentation system.

Table 1. Percentage of clinical cases in patients with diabetic ulcers

\begin{tabular}{lccc}
\hline \multicolumn{2}{l}{ Clinical cases [n=150, (\%)] } & $\begin{array}{c}\text { Male } \\
{[n=119,(\%)]}\end{array}$ & $\begin{array}{c}\text { Female } \\
{[n=31(\%)]}\end{array}$ \\
\hline DFU & $106(73.33)$ & $85(71.42)$ & $21(67.74)$ \\
Gangrene & $24(16)$ & $17(14.28)$ & $7(22.58)$ \\
Accidental & $9(6)$ & $7(5.88)$ & $2(6.45)$ \\
Burns & $7(4.66)$ & $6(5.04)$ & $1(3.22)$ \\
\hline
\end{tabular}

\section{RESULTS}

A total of 150 patients with diabetic ulcers were enrolled. Most of them developed diabetic foot ulcers; whereas few patients developed gangrene, some also developed ulcers after an accident or burns. Out of the total patients studied 106 (73\%) people developed diabetic foot ulcers; whereas 24 (16\%) patients developed gangrene due to loss of blood supplies to the extremities, 9 (6\%) people suffered from ulcers accidentally and only 7 (4.6\%) people developed ulcers after burns. Further 85 (71\%) males and 21 (67\%) females developed DFUs whereas; 7 (22\%) females and 17 (14\%) males developed gangrene. About 5\% each of males suffered from DFUs either due to accident and/or burns (Table-1 and Fig. 1). As per the age group wise distribution with signs and

Table 2. Prevalence of age group and Sepsis patient related to diabetic foot ulcer

\begin{tabular}{lc}
\hline Bacterial isolates & $\begin{array}{c}\text { No. of Diabetic Ulcer } \\
\text { Patients [ } \mathrm{n}=150(\%)]\end{array}$ \\
\hline S.aureus & $78(52)$ \\
S.aureus + P.aeruginosa & $15(10)$ \\
S.aureus + E.coli & $21(14)$ \\
P.aeruginosa & $19(12.66)$ \\
P.aeruginosa + E.coli & $6(4)$ \\
P.aeruginosa + Klebsiella sp & $2(1.33)$ \\
E.coli & $6(4)$ \\
E.coli + Klebsiella sp & $1(0.66)$ \\
Klebsiella sp & $2(1.33)$ \\
\hline
\end{tabular}
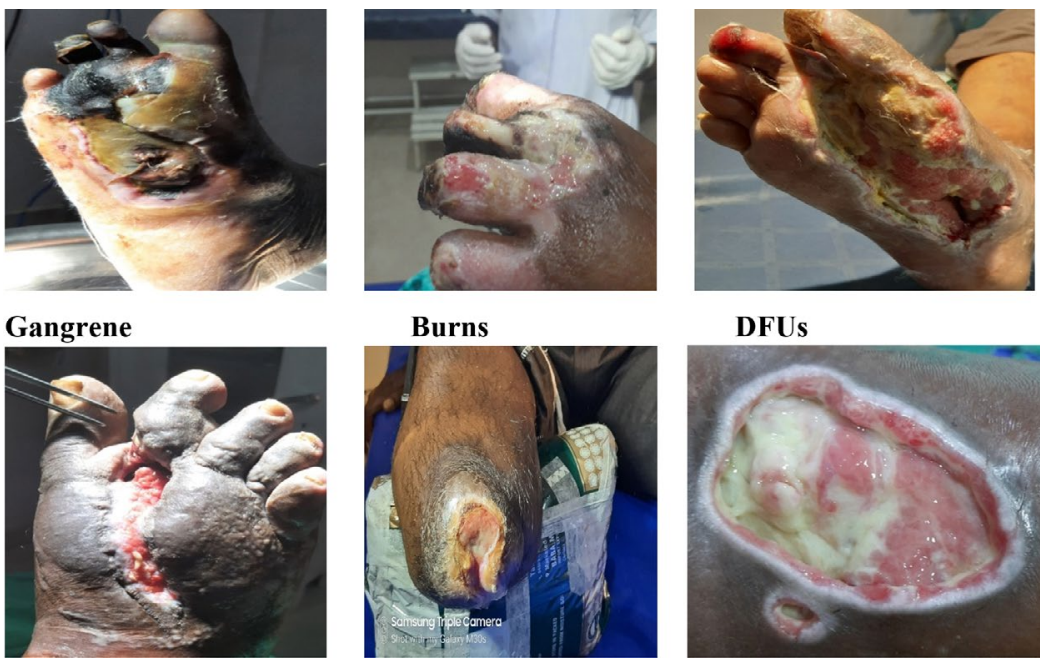

Fig. 1. Different types of ulcers in patients with diabetes (Gangrene, DFUs, Burn cases, Accidental cases and Amputation cases). 
symptoms of patients, $119(79.4 \%)$ were males and $31(20.6 \%)$ were females. $112(74.6 \%)$ patients in the age group $\geq 45-55$ years and $34(22 \%)$ in the age group $\geq 55-65$ years developed DFUs. About $105(70 \%)$ of patients had infection from single bacterial species; whereas 45 (30\%) patients had polymicrobial infection. In this study, different age groups, duration of diabetes, types of infection, duration of infection and medication were the significant risk factors in DFUs (Table 2). The microbiological profile of samples from patients with diabetic foot ulcers showed a total number of 195 isolates were detected from 150 ulcer specimens. S.aureus was isolated from 114 (76\%) of the samples followed by 42 (28\%) Pseudomonas aeruginosa. E.coli was found 34 (22\%) of the samples. The infection status of samples from patients with diabetic foot ulcers.78 (52\%) of the samples were infected with S.aureus, 19 (12\%) with P.aeruginosa, 6 (4\%) with E.coli whereas; 2 (1\%) had Klebsiella spp. only (Table-3). It was found that the major etiological agent was S.aureus followed by P.aeruginosa and E.coli. S.aureus was one of the most important microorganism that manifests a range of clinical problems resulting from high-resistance to anti-microbial agents. PCR was performed for the confirmation of S.aureus. All the samples were positive for nucA gene which confirmed the presence of S.aureus in diabetic ulcer patients. (Fig. 2)

Table 3. Bacterial etiology related to diabetic ulcer patients

\begin{tabular}{lll}
\hline Parameters & & $\begin{array}{l}\text { No. of patients with } \\
\text { Diabetic Ulcers } \\
\mathrm{n}=150,(\%)\end{array}$ \\
\hline $\begin{array}{lll}\text { Age Group } \\
\text { (in years) }\end{array}$ & $>30-45$ & $2(1.33 \%)$ \\
& $>60$ & $138(92 \%)$ \\
Duration of & $<10$ & $10(6.66 \%)$ \\
Diabetes & $>10$ & $57(38 \%)$ \\
(in years) & & $93(62 \%)$ \\
Types of & Mono-microbial & $105(70)$ \\
Infection & Poly-microbial & $45(30)$ \\
Duration of & $1-9$ & $71(55.33 \%)$ \\
Infection & $10-19$ & $72(40 \%)$ \\
(in months) & $20-29$ & $4(2.66 \%)$ \\
& $\geq 30$ & $3(2 \%)$ \\
Medication & Insulin & $74(49.33 \%)$ \\
& Oral & $76(50.66 \%)$ \\
\hline
\end{tabular}

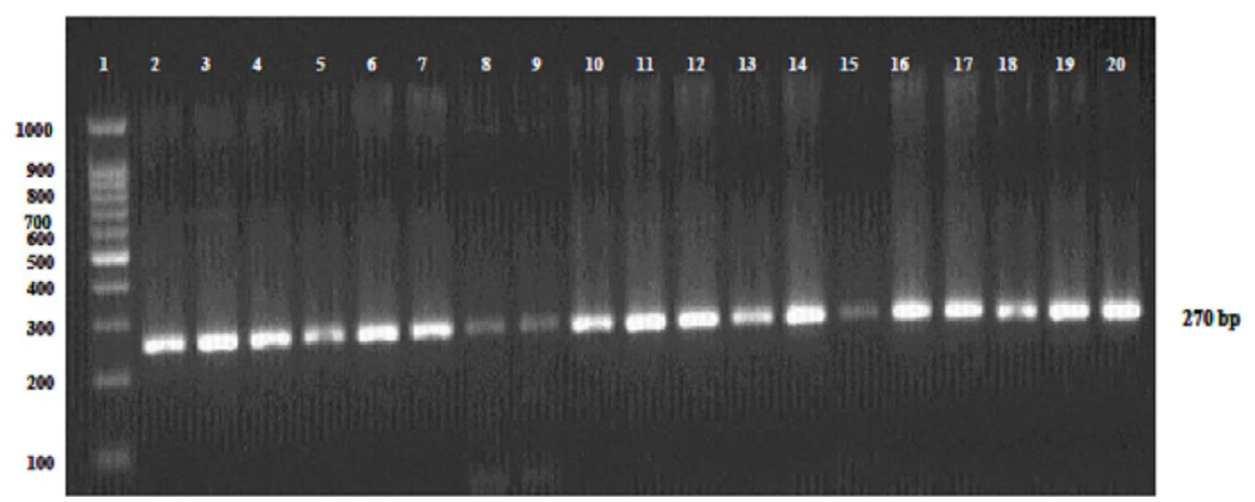

Fig. 2. Agarose gel electrophoresis showing PCR amplification of nucA gene of $S$. aureus isolates. [Lane 1:100bp ladder, lane2-19 S.aureus DNA, lane 20: ATCC strain of S. aureus (25923)].

\section{DISCUSSION}

It was observed that S.aureus was the major etiological agent among one hundred fifty diabetic ulcers samples, S.aureus were concomitantly isolated from diabetic foot ulcer patients. Our finding is consistent with previous studies reported by Mottola et $\mathrm{al}^{10}$. S.aureus is one of the most important micro-organisms that cause clinical problems resulting high-resistance to different antimicrobial agents. Though it is rarely found in the normal flora of humans, it is frequently isolated from patients with burns, cystic fibrosis and neutropenia ${ }^{11}$. Diabetic foot ulcer is one of the most common devastating complications of diabetes mellitus and the leading cause of agonizing amputation throughout the world $^{12}$. These infections may be colonized by pathogenic and anti-microbial resistant bacteria, 
harbouring several virulence factors that could impair its successful treatment ${ }^{13}$. Certain sociocultural practices in India like barefoot walking, poor hygiene habits, inadequate facilities for diabetic care, low level of education and poor socio-economic conditions often lead to foot lesions and hospitalizations ${ }^{14}$. The present study showed $80 \%$ male ascendancy followed by $20 \%$ female. However, the present study is also comparable with a multicentric study from Saudi Arabia ${ }^{15,16}$. Direct antibiotic treatment cannot be recommended as per clinical signs or symptoms of infection as there is a very fine line between colonization of micro-organisms and problematic bio-burden and the direct antibiotic treatment efficacy remains unclear on the basis of only these two major determinants. Whereas, treatment options based upon targeting microbial population to promote healing and determining infection related complications might be a novel one $^{17}$. S. aureus may cause severe tissue damage in diabetic patients and should never be ignored as insignificant in diabetic foot ulcers. Moreover, it should never be considered a contaminant or normal flora, and it should clearly be considered a pathogen, because it may result in sepsis and amputation ${ }^{18}$. The numerous virulence factors and toxins secreted by S.aureus during infection that evade host immune defences are few of the challenges in managing S.aureus infections is an inherent resistance mechanism, referred to as intrinsic resistance. Its multiplicity of resistance mechanisms may render this microbe less amenable to control by antibiotic cycling. S.aureus is noted for its metabolic versatility and its exceptional ability to colonize a wide variety of environments and also for its intrinsic resistance to a wide variety of antimicrobial agent ${ }^{19}$.

There are many studies that suggest that apart from clinical factors, the socio-demographic variables play an important role in diabetic foot ulceration. It was reported by DeBerardis et al (2005) that the prevalence of diabetic foot complications was higher in older patients, those with limited formal education and a low socio-demographic status ${ }^{20}$. Our results couldn't substantiate the claim mentioned in the above study. One ninety five micro-organisms were isolated from one fifty clinical samples of diabetic foot ulcers, which showed multiple bacterial infections that represent an average of 1.3 organisms per ulcer which is slightly lower than other studies ${ }^{21,22}$, and showed an average of 1.52 organisms per ulcer. Diabetic foot infections are usually polymicrobial in nature and this has been well documented in the literature. S.aureus was the most common isolate observed in diabetic foot ulcers that was in accordance with findings of previous studies ${ }^{23,24}$. The emergence of $S$.aureus in the DFUs caused severe wound infection and worsened of the wound ${ }^{25}$. However, the bacterial diversity and prevalence of specific bacteria vary greatly from studies to studies. Identification of diabetes with DFUs and its associated factors are the key to reduce further complications and to have baseline information to initiate appropriate interventions.

\section{CONCLUSION}

The present study reports that S.aureus was the major etiological agent with sociodemographic and clinical profile of patients with diabetic ulcer. There was a predominance of monomicrobial growth with gram-positive organisms. Healthcare should be made more accessible to facilitate early diagnosis of DFU and its complications to minimize the rate of amputations. This type of study should be continued for a longer period both in coastal and tribal areas of Odisha.

\section{ACKNOWLEDGMENTS}

Thanks to ICMR, NEW DELHI, [NO.2019

$-4588 / \mathrm{NCD}-\mathrm{III}]$, for their support.

\section{CONFLICT OF INTEREST}

The authors declare that there is no conflict of interest.

\section{AUTHORS' CONTRIBUTION}

All authors listed have made a substantial, direct and intellectual contribution to the work, and approved it for publication.

\section{FUNDING}

The project was funded by ICMR-NEW DELHI [NO.2019-4588/NCD-III] 


\section{DATA AVAILABILITY}

All datasets generated or analyzed during this study are included in the manuscript.

\section{ETHICS STATEMENT}

The study was approved by the institutional human ethics committee of the ICMRRegional medical research centre, Bhubaneswar and was carried out in accordance with the approved guidelines.

\section{REFERENCES}

1. International Diabetes Federation. IDF DIABETES ATLAS. Ninth edition 2019.

2. Enoch $\mathrm{S}$, Harding $\mathrm{K}$, Wound bed preparation: the science behind the removal of barriers to healing. Wounds. 2003;15(7):213-229.

3. Gadepalli R, Dhawan B, Sreenivas V, Kapil A, Ammini AC, Chaudhry R. A Clinico microbiological study of diabetic foot ulcers in an Indian tertiary care hospital. Diabetes Care. 2006;29(8):1727-1732. doi: 10.2337/ dc06-0116

4. Shankhdhar K, Shankhdhar LK, Shankhdhar U, Shankhdhar S. Diabetic foot problems in India: an overview and potential simple approaches in a developing country. Curr Diab Rep. 2008;8(6):452-457. doi: 10.1007/s11892-008-0078-y

5. Chun DI, Kim S, Kim J, et al. Epidemiology and burden of diabetic foot ulcer and peripheral arterial disease in Korea. J Clin Med. 2019;8(5):748. doi: 10.3390/ jcm8050748

6. Gardner SE, Frantz RA, Doebbeling BN. The validity of the clinical signs and symptoms used to identify localized chronic wound infection. Wound Repair and Regen. 2001;9(3):178-186. doi: 10.1046/j.1524475x.2001.00178.x

7. Singh N, Armstrong DG, Lipsky BA. Preventing foot ulcer in patients with diabetes. JAMA. 2005;293(2):217-228. doi: 10.1001/jama.293.2.217

8. Standard operating procedures Bacteriology. 1st Edition. Antimicrobial Resistance Surveillance and Research Network, ICMR. 2015;40-43.

9. Sambrook J, Fritsch EF, Maniatis T. Molecular cloning: a laboratory manual. Cold spring harbor laboratory. 1989.

10. Mottola C, Matias CS, Mendes JJ, et al. Susceptibility patterns of Staphylococcus aureus bio-films in diabetic foot infections. BMC Microbiology. 2016;16(1):119. doi: 10.1186/s12866-016-0737-0

11. Zubair M, Malik A, Ahmad J. Incidence, risk factors for amputation among patients with diabetic foot ulcer in a North Indian tertiary care hospital. The Foot. 2012;22(1):24-30. doi: 10.1016/j.foot.2011.09.003

12. Roberts AD, Simon GL. Diabetic foot infections: the role of microbiology and antibiotic treatment. Seminars in Vasc Surg. 2012;25(2,):75-81. doi: 10.1053/j. semvascsurg.2012.04.010

13. Dunyach-Remy C, Ngba Essebe C, Sotto A, Lavigne J-P. Staphylococcus aureus toxins and diabetic foot ulcers: role in pathogenesis and interest in diagnosis. Toxins. 2016;8(7):209. doi: 10.3390/toxins8070209

14. Shahi SK, Kumar A, Kumar S, Singh SK, Gupta SK, Singh TB. Prevalence of diabetic foot ulcer and associated risk factors in diabetic patients from North India. $J$ Diabetic Foot Complication. 2012;4(3):83-91.

15. Viswanathan V, Thomas N, Tandon N, Asirvatham A, Rajasekar S. Profile of diabetic foot complications and its associated complications-a multi-centric study from India. JAPI. 2005;53:933-936.

16. Al-Rubeaan K, Al Derwish M, Ouizi S, et al. Diabetic foot complications and their risk factors from a large retrospective cohort study. PloS One. $2015 ; 10(5)$ :e124446 doi: 10.1371/journal. pone.0124446

17. Lipsky BA, Aragon-Sanchez J, Diggle M, et al. IWGDF guidance on the diagnosis and management of foot infections in persons with diabetes. Diabetes Metab Res Rev. 2016;32(Suppl. 1):45-74. doi: 10.1002/ dmrr.2699

18. Jyothylekshmy V, Menon AS, Abraham S. Epidemiology of diabetic foot complications in a podiatry clinic of a tertiary hospital in South India. Indian Journal of Health Sciences and Biomedical Research (KLEU). 2015;8(1):48-51. doi: 10.4103/2349-5006.158231

19. Sangeeta J, Ray P, Manchanda V, et al. Methicillin resistant Staphylococcus aureus (MRSA) in India: prevalence \& susceptibility pattern. Indian J Med Res. 2013;137(2):363-369.

20. De Berardis G, Pellegrini F, Franciosi M, et al. Are Type 2 diabetic patients offered adequate foot care? The role of physician and patient characteristics. J Diabetes Complications. 2005;19(6):319-327. doi: 10.1016/j. jdiacomp.2005.02.005

21. Raja NS. Microbiology of diabetic foot infections in a teaching hospital in Malaysia: a retrospective study of 194 cases. J Microbiol Immunol Infect. 2007;40(1):3944.

22. Bansal E, Garg A, Bhatia S, Attri AK, Chander J. Spectrum of microbial flora in diabetic foot ulcers. Indian J Pathol Microbiol. 2008;51(2):204-208. doi: 10.4103/03774929.41685

23. Abdulrazak A, Bitar ZI, Al-Shamali AA, Mobasher LA. Bacteriological study of diabetic foot infections. J Diabetes Complications. 2005;19(3):138-141. doi: 10.1016/j.jdiacomp.2004.06.001

24. Sharma VK, Khadka PB, Joshi A, Sharma R. Common pathogens isolated in diabetic foot infection in Bir Hospital. Kathmandu Univ Med J (KUMJ). 2006;4(3):295-301.

25. Shettigar K, Murali TS. Virulence factors and clonal diversity of Staphylococcus aureus in colonization and wound infection with emphasis on diabetic foot infection. European Eur J Clin Microbiol Infect Dis. 2020;39:2235-2246. doi: 10.1007/s10096-020-039848 